\title{
Some Fixed Point Theorems in $G$-metric Spaces
}

\author{
Sushanta Kumar Mohanta
}

\begin{abstract}
We prove some fixed point theorems for self mappings satisfying some kind of contractive type conditions on complete $G$-metric spaces.
\end{abstract}

\section{Introduction}

The study of metric fixed point theory plays an important role because the study finds applications in many important areas as diverse as differential equations, operation research, mathematical economics and the like. Different generalizations of the usual notion of a metric space were proposed by several mathematicians such as Gähler $[4,5]$ (called 2-metric spaces) and Dhage $[2,3]$ (called $D$-metric spaces). K.S.Ha et.al. [6] have pointed out that the results cited by Gähler are independent, rather than generalizations, of the corresponding results in metric spaces. Moreover, it was shown that Dhage's notion of $D$-metric space is flawed by errors and most of the results established by him and others are invalid. These facts determined Mustafa and Sims [12] to introduce a new concept in the area, called $G$-metric space. Recently, Mustafa et.al. studied many fixed point theorems for mappings satisfying various contractive conditions on complete $G$-metric spaces; see [9-15]. Subsequently, some authors like Renu Chugh et.al.[1], W. Shatanawi [17] have generalized some results of Mustafa et.al. [9, 10] and studied some fixed point results for self-mapping in a complete $G$-metric space under some contractive conditions related to a non-decreasing map $\phi:[0,+\infty) \rightarrow[0,+\infty)$ with $\lim _{n \rightarrow \infty} \phi^{n}(t)=0$

Key Words: $G$-metric space, $G$-Cauchy sequence, fixed point.

2010 Mathematics Subject Classification: 54H25, 54C60.

Received: December, 2010.

Revised: March, 2011.

Accepted: February, 2012. 
for all $t \in(0,+\infty)$. In the present work, we prove some fixed point theorems for self-mappings on complete $G$-metric spaces. Our results generalize some recent results in the setting of $G$-metric space.

\section{Definitions and Preliminaries}

We begin by briefly recalling some basic definitions and results for $G$-metric spaces that will be needed in the sequel.

Definition 2.1. (see [8]) Let $X$ be a non empty set, and let $G: X \times X \times$ $X \rightarrow R^{+}$be a function satisfying the following axioms:

$$
\begin{aligned}
& \left(G_{1}\right) G(x, y, z)=0 \text { if } x=y=z, \\
& \left(G_{2}\right) 0<G(x, x, y), \text { for all } x, y \in X, \text { with } x \neq y, \\
& \left(G_{3}\right) G(x, x, y) \leq G(x, y, z), \text { for all } x, y, z \in X, \text { with } z \neq y \\
& \left(G_{4}\right) G(x, y, z)=G(x, z, y)=G(y, z, x)=\cdots(\text { symmetry in all three variables }), \\
& \left.\left(G_{5}\right) G(x, y, z) \leq G(x, a, a)+G(a, y, z), \text { for all } x, y, z, a \in X, \text { (rectangle inequality }\right) .
\end{aligned}
$$

Then the function $G$ is called a generalized metric, or, more specifically a $G$-metric on $X$, and the pair $(X, G)$ is called a $G$-metric space.

Example 2.2. (see [8]) Let $R$ be the set of all real numbers. Define $G$ : $R \times R \times R \rightarrow R^{+}$by

$$
G(x, y, z)=|x-y|+|y-z|+|z-x|, \text { for all } x, y, z \in X .
$$

Then it is clear that $(R, G)$ is a $G$-metric space.

Proposition 2.3. (see [8]) Let $(X, G)$ be a $G$-metric space. Then for any $x, y, z$, and $a \in X$, it follows that

(1) if $G(x, y, z)=0$ then $x=y=z$,

(2) $G(x, y, z) \leq G(x, x, y)+G(x, x, z)$,

(3) $G(x, y, y) \leq 2 G(y, x, x)$,

(4) $G(x, y, z) \leq G(x, a, z)+G(a, y, z)$,

(5) $G(x, y, z) \leq \frac{2}{3}(G(x, y, a)+G(x, a, z)+G(a, y, z))$, 
(6) $G(x, y, z) \leq G(x, a, a)+G(y, a, a)+G(z, a, a)$.

Definition 2.4. (see [8]) Let $(X, G)$ be a $G$-metric space, let $\left(x_{n}\right)$ be a sequence of points of $X$, we say that $\left(x_{n}\right)$ is $G$-convergent to $x$ if $\lim _{n, m \rightarrow \infty} G\left(x, x_{n}, x_{m}\right)=$ 0 ; that is , for any $\epsilon>0$, there exists $n_{0} \in N$ such that $G\left(x, x_{n}, x_{m}\right)<$ $\epsilon$, for all $n, m \geq n_{0}$. We refer to $x$ as the limit of the sequence $\left(x_{n}\right)$ and write $x_{n} \stackrel{(G)}{\longrightarrow} x$.

Proposition 2.5. (see [8]) Let $(X, G)$ be a $G$-metric space. Then, the following are equivalent:

(1) $\left(x_{n}\right)$ is G-convergentto $x$.

(2) $G\left(x_{n}, x_{n}, x\right) \rightarrow 0$, as $n \rightarrow \infty$.

(3) $G\left(x_{n}, x, x\right) \rightarrow 0$, as $n \rightarrow \infty$.

(4) $G\left(x_{m}, x_{n}, x\right) \rightarrow 0$, as $m, n \rightarrow \infty$.

Definition 2.6. (see [8]) Let $(X, G)$ be a $G$-metric space, a sequence $\left(x_{n}\right)$ is called $G$-Cauchy if given $\epsilon>0$, there is $n_{0} \in N$ such that $G\left(x_{n}, x_{m}, x_{l}\right)<$ $\epsilon$, for all $n, m, l \geq n_{0}$ that is if $G\left(x_{n}, x_{m}, x_{l}\right) \rightarrow 0$ as $n, m, l \rightarrow \infty$.

Proposition 2.7. (see [8]) In a $G$-metric space $(X, G)$, the following are equivalent.

(1) The sequence $\left(x_{n}\right)$ is G-Cauchy.

(2) For every $\epsilon>0$, there exists $n_{0} \in N$ such that $G\left(x_{n}, x_{m}, x_{m}\right)<\epsilon$ for all $n, m \geq n_{0}$.

Definition 2.8. (see [8]) Let $(X, G)$ and $\left(X^{\prime}, G^{\prime}\right)$ be $G$-metric spaces and let $f:(X, G) \rightarrow\left(X^{\prime}, G^{\prime}\right)$ be a function, then $f$ is said to be $G$-continuous at a point $a \in X$ if given $\epsilon>0$, there exists $\delta>0$ such that $x, y \in X ; G(a, x, y)<$ $\delta$ implies $G^{\prime}(f(a), f(x), f(y))<\epsilon$. A function $f$ is $G$-continuous on $X$ if and only if it is $G$-continuous at all $a \in X$.

Proposition 2.9. (see [8]) Let $(X, G)$ and $\left(X^{\prime}, G^{\prime}\right)$ be $G$-metric spaces, then a function $f: X \rightarrow X^{\prime}$ is $G$-continuous at a point $x \in X$ if and only if it is $G$-sequentially continuous at $x$; that is, whenever $\left(x_{n}\right)$ is $G$-convergent to $x,\left(f\left(x_{n}\right)\right)$ is $G$-convergent to $f(x)$.

Proposition 2.10. (see [8]) Let $(X, G)$ be a $G$-metric space. Then, the function $G(x, y, z)$ is continuous in all variables.

Definition 2.11. (see [8]) A $G$-metric space $(X, G)$ is said to be $G$-complete (or a complete $G$-metric space) if every $G$-Cauchy sequence in $(X, G)$ is $G$ convergent in $(X, G)$. 


\section{Main Results}

In this section we present some fixed point theorems for self mappings satisfying various contractive conditions on complete $G$-metric spaces.

Theorem 3.1. Let $(X, G)$ be a complete $G$-metric space, and let $T: X \longrightarrow$ $X$ be such that

$$
\begin{aligned}
G(T(x), T(y), T(z)) \leq & a G(x, y, z)+b G(x, T(x), T(x)) \\
& +c G(y, T(y), T(y))+d G(z, T(z), T(z)) \\
+ & +\max \left\{\begin{array}{c}
G(x, T(y), T(y)), G(y, T(x), T(x)), \\
G(y, T(z), T(z)), G(z, T(y), T(y)), \\
G(z, T(x), T(x)), G(x, T(z), T(z))
\end{array}\right\}
\end{aligned}
$$

for all $x, y, z \in X$, where $a, b, c, d, e \geq 0$ with $a+b+c+d+2 e<1$. Then $T$ has a unique fixed point (say $u$ ) in $X$ and $T$ is $G$-continuous at $u$.

Proof. Let $x_{0} \in X$ be an arbitrary point and define the sequence $\left(x_{n}\right)$ by $x_{n}=T^{n}\left(x_{0}\right)$. Assume $x_{n} \neq x_{n+1}$ for all $n$. Then by (1), we have

$$
\begin{aligned}
G\left(x_{n}, x_{n+1}, x_{n+1}\right) \leq & a G\left(x_{n-1}, x_{n}, x_{n}\right)+b G\left(x_{n-1}, x_{n}, x_{n}\right) \\
& +c G\left(x_{n}, x_{n+1}, x_{n+1}\right)+d G\left(x_{n}, x_{n+1}, x_{n+1}\right) \\
+ & +\max \left\{\begin{array}{l}
G\left(x_{n-1}, x_{n+1}, x_{n+1}\right), G\left(x_{n}, x_{n}, x_{n}\right), \\
G\left(x_{n}, x_{n+1}, x_{n+1}\right), G\left(x_{n}, x_{n+1}, x_{n+1}\right), \\
G\left(x_{n}, x_{n}, x_{n}\right), G\left(x_{n-1}, x_{n+1}, x_{n+1}\right)
\end{array}\right\} .
\end{aligned}
$$

So, it must be the case that,

$$
G\left(x_{n}, x_{n+1}, x_{n+1}\right) \leq\left\{\begin{array}{l}
a G\left(x_{n-1}, x_{n}, x_{n}\right)+b G\left(x_{n-1}, x_{n}, x_{n}\right) \\
+c G\left(x_{n}, x_{n+1}, x_{n+1}\right)+d G\left(x_{n}, x_{n+1}, x_{n+1}\right) \\
+e \max \left\{G\left(x_{n-1}, x_{n+1}, x_{n+1}\right), G\left(x_{n}, x_{n+1}, x_{n+1}\right)\right\}
\end{array}\right\} .
$$

But by $\left(G_{5}\right)$, we have

$$
G\left(x_{n-1}, x_{n+1}, x_{n+1}\right) \leq G\left(x_{n-1}, x_{n}, x_{n}\right)+G\left(x_{n}, x_{n+1}, x_{n+1}\right) .
$$


So, (2) becomes

$$
\begin{aligned}
G\left(x_{n}, x_{n+1}, x_{n+1}\right) \leq & a G\left(x_{n-1}, x_{n}, x_{n}\right)+b G\left(x_{n-1}, x_{n}, x_{n}\right)+c G\left(x_{n}, x_{n+1}, x_{n+1}\right) \\
& +d G\left(x_{n}, x_{n+1}, x_{n+1}\right)+e\left\{G\left(x_{n-1}, x_{n}, x_{n}\right)+G\left(x_{n}, x_{n+1}, x_{n+1}\right)\right\}
\end{aligned}
$$

which implies that,

$$
G\left(x_{n}, x_{n+1}, x_{n+1}\right) \leq \frac{a+b+e}{1-c-d-e} G\left(x_{n-1}, x_{n}, x_{n}\right) .
$$

Let $q=\frac{a+b+e}{1-c-d-e}$, then $q<1$ since $a+b+c+d+2 e<1$ and by repeated application of $(3)$, we have

$$
G\left(x_{n}, x_{n+1}, x_{n+1}\right) \leq q^{n} G\left(x_{0}, x_{1}, x_{1}\right) .
$$

Then, for all $n, m \in N, n<m$, we have by repeated use of the rectangle inequality and (4) that

$$
\begin{aligned}
G\left(x_{n}, x_{m}, x_{m}\right) \leq & G\left(x_{n}, x_{n+1}, x_{n+1}\right)+G\left(x_{n+1}, x_{n+2}, x_{n+2}\right) \\
& +G\left(x_{n+2}, x_{n+3}, x_{n+3}\right)+\cdots+G\left(x_{m-1}, x_{m}, x_{m}\right) \\
\leq & \left(q^{n}+q^{n+1}+\cdots+q^{m-1}\right) G\left(x_{0}, x_{1}, x_{1}\right) \\
\leq & \frac{q^{n}}{1-q} G\left(x_{0}, x_{1}, x_{1}\right) .
\end{aligned}
$$

Then, $\lim G\left(x_{n}, x_{m}, x_{m}\right)=0$, as $n, m \rightarrow \infty$, since $\lim \frac{q^{n}}{1-q} G\left(x_{0}, x_{1}, x_{1}\right)=0$, as $n, m \rightarrow \infty$. For $n, m, l \in N,\left(G_{5}\right)$ implies that

$$
G\left(x_{n}, x_{m}, x_{l}\right) \leq G\left(x_{n}, x_{m}, x_{m}\right)+G\left(x_{l}, x_{m}, x_{m}\right),
$$

taking limit as $n, m, l \rightarrow \infty$, we get $G\left(x_{n}, x_{m}, x_{l}\right) \rightarrow 0$. So $\left(x_{n}\right)$ is a $G$-Cauchy sequence. By completeness of $(X, G)$, there exists $u \in X$ such that $\left(x_{n}\right)$ is $G$-convergent to $u$. Suppose that $T(u) \neq u$, then

$$
\begin{aligned}
G\left(x_{n}, T(u), T(u)\right) \leq & a G\left(x_{n-1}, u, u\right)+b G\left(x_{n-1}, x_{n}, x_{n}\right) \\
& +c G(u, T(u), T(u))+d G(u, T(u), T(u)) \\
+ & +\max \left\{\begin{array}{c}
G\left(x_{n-1}, T(u), T(u)\right), G\left(u, x_{n}, x_{n}\right), \\
G(u, T(u), T(u)), G(u, T(u), T(u)), \\
G\left(u, x_{n}, x_{n}\right), G\left(x_{n-1}, T(u), T(u)\right)
\end{array}\right\} .
\end{aligned}
$$

Taking the limit as $n \rightarrow \infty$, and using the fact that the function $G$ is continuous on its variables, we have

$$
G(u, T(u), T(u)) \leq(c+d+e) G(u, T(u), T(u)),
$$


which is a contradiction since $0 \leq c+d+e<1$. So, $u=T(u)$.

For uniqueness of $u$, suppose that $v \neq u$ is such that $T(v)=v$, then (1) implies that

$$
\begin{aligned}
G(u, v, v)=G(T(u), T(v), T(v)) \leq & a G(u, v, v)+b G(u, u, u)+c G(v, v, v) \\
& +d G(v, v, v)+e \max \left\{\begin{array}{c}
G(u, v, v), G(v, u, u), \\
G(v, v, v), G(v, v, v), \\
G(v, u, u), G(u, v, v)
\end{array}\right\} .
\end{aligned}
$$

So, it must be the case that,

$$
\begin{aligned}
G(u, v, v) & \leq a G(u, v, v)+e \max \{G(u, v, v), G(v, u, u)\} \\
& \leq a G(u, v, v)+e \max \{2 G(v, u, u), G(v, u, u)\} \\
& =a G(u, v, v)+2 e G(v, u, u) .
\end{aligned}
$$

Thus,

$$
G(u, v, v) \leq \frac{2 e}{1-a} G(v, u, u) .
$$

Again by the same argument we will find

$$
G(v, u, u) \leq \frac{2 e}{1-a} G(u, v, v) .
$$

Thus, we have

$$
G(u, v, v) \leq\left(\frac{2 e}{1-a}\right)^{2} G(u, v, v)
$$

which implies that, $u=v$, since $0 \leq \frac{2 e}{1-a}<1$.

To see that $T$ is $G$-continuous at $u$, let $\left(y_{n}\right)$ be any sequence in $X$ such that $\left(y_{n}\right)$ is $G$-convergent to $u$. For $n \in N$, we have

$$
\begin{aligned}
G\left(T\left(y_{n}\right), T(u), T\left(y_{n}\right)\right) \leq & a G\left(y_{n}, u, y_{n}\right)+b G\left(y_{n}, T\left(y_{n}\right), T\left(y_{n}\right)\right) \\
& +c G(u, u, u)+d G\left(y_{n}, T\left(y_{n}\right), T\left(y_{n}\right)\right) \\
& +e \max \left\{\begin{array}{l}
G\left(y_{n}, u, u\right), G\left(u, T\left(y_{n}\right), T\left(y_{n}\right)\right), \\
G\left(u, T\left(y_{n}\right), T\left(y_{n}\right)\right), G\left(y_{n}, u, u\right), \\
G\left(y_{n}, T\left(y_{n}\right), T\left(y_{n}\right)\right), G\left(y_{n}, T\left(y_{n}\right), T\left(y_{n}\right)\right)
\end{array}\right\} .
\end{aligned}
$$


But, $G\left(y_{n}, T\left(y_{n}\right), T\left(y_{n}\right)\right) \leq G\left(y_{n}, u, u\right)+G\left(u, T\left(y_{n}\right), T\left(y_{n}\right)\right)$.

Thus,

$$
\begin{aligned}
G\left(T\left(y_{n}\right), T(u), T\left(y_{n}\right)\right) \leq \quad & a G\left(y_{n}, y_{n}, u\right)+(b+d)\left\{G\left(y_{n}, u, u\right)+G\left(u, T\left(y_{n}\right), T\left(y_{n}\right)\right)\right\} \\
& +e\left\{G\left(y_{n}, u, u\right)+G\left(u, T\left(y_{n}\right), T\left(y_{n}\right)\right)\right\}
\end{aligned}
$$

which gives,

$$
G\left(u, T\left(y_{n}\right), T\left(y_{n}\right)\right) \leq \frac{b+d+e}{1-b-d-e} G\left(y_{n}, u, u\right)+\frac{a}{1-b-d-e} G\left(y_{n}, y_{n}, u\right)
$$

Taking the limit as $n \rightarrow \infty$, we see that $G\left(u, T\left(y_{n}\right), T\left(y_{n}\right)\right) \rightarrow 0$ and so, by Proposition 2.5, the sequence $\left(T\left(y_{n}\right)\right)$ is $G$-convergent to $u=T(u)$. Therefore Proposition 2.9 implies that $T$ is $G$-continuous at $u$.

As an application of Theorem 3.1, we have the following Corollary.

Corollary 3.2. Let $(X, G)$ be a complete $G$-metric space, and let $T: X \longrightarrow$ $X$ satisfies for some $m \in N$ :

$$
\begin{aligned}
& G\left(T^{m}(x), T^{m}(y), T^{m}(z)\right) \leq a G(x, y, z)+b G\left(x, T^{m}(x), T^{m}(x)\right) \\
& +c G\left(y, T^{m}(y), T^{m}(y)\right)+d G\left(z, T^{m}(z), T^{m}(z)\right) \\
& +e \max \left\{\begin{array}{c}
G\left(x, T^{m}(y), T^{m}(y)\right), G\left(y, T^{m}(x), T^{m}(x)\right), \\
G\left(y, T^{m}(z), T^{m}(z)\right), G\left(z, T^{m}(y), T^{m}(y)\right), \\
G\left(z, T^{m}(x), T^{m}(x)\right), G\left(x, T^{m}(z), T^{m}(z)\right)
\end{array}\right\}
\end{aligned}
$$

for all $x, y, z \in X$, where $a, b, c, d, e \geq 0$ with $a+b+c+d+2 e<1$. Then $T$ has a unique fixed point (say $u$ ) in $X$ and $T^{m}$ is G-continuous at $u$.

Proof. From Theorem 3.1, we see that $T^{m}$ has a unique fixed point (say $u$ ) in $X$ and $T^{m}$ is $G$-continuous at $u$. Since

$$
T(u)=T\left(T^{m}(u)\right)=T^{m+1}(u)=T^{m}(T(u)),
$$

we have that $T(u)$ is also a fixed point for $T^{m}$. By uniqueness of $u$, we get $T(u)=u$.

Remark 3.3. We see that Theorem 3.1 is generalization of the Result [10, Theorem 2.1], where [10, Theorem 2.1] obtained by taking $e=0$ in Theorem 3.1 . 
Theorem 3.4. Let $(X, G)$ be a complete $G$-metric space, and let $T: X \longrightarrow$ $X$ be such that

$$
\begin{aligned}
G(T(x), T(y), T(z)) \leq & a\{G(x, T(y), T(y))+G(y, T(x), T(x))\} \\
& +b\{G(y, T(z), T(z))+G(z, T(y), T(y))\} \\
& +c\{G(z, T(x), T(x))+G(x, T(z), T(z))\}+d G(x, y, z) \\
& +e \max \{G(x, T(x), T(x)), G(y, T(y), T(y)), G(z, T(z), T(z))\}
\end{aligned}
$$

for all $x, y, z \in X$, where $a, b, c, d, e \geq 0$ with $2 a+2 b+2 c+d+2 e<1$. Then $T$ has a unique fixed point (say $u$ ) in $X$ and $T$ is $G$-continuous at $u$.

Proof. Let $x_{0} \in X$ be an arbitrary point and define the sequence $\left(x_{n}\right)$ by $x_{n}=T^{n}\left(x_{0}\right)$. Assume $x_{n} \neq x_{n+1}$ for all $n$. Then by (5), we have

$$
\begin{aligned}
G\left(x_{n}, x_{n+1}, x_{n+1}\right) \leq & a\left\{G\left(x_{n-1}, x_{n+1}, x_{n+1}\right)+G\left(x_{n}, x_{n}, x_{n}\right)\right\} \\
& +b\left\{G\left(x_{n}, x_{n+1}, x_{n+1}\right)+G\left(x_{n}, x_{n+1}, x_{n+1}\right)\right\} \\
& +c\left\{G\left(x_{n}, x_{n}, x_{n}\right)+G\left(x_{n-1}, x_{n+1}, x_{n+1}\right)\right\}+d G\left(x_{n-1}, x_{n}, x_{n}\right) \\
& +e \max \left\{G\left(x_{n-1}, x_{n}, x_{n}\right), G\left(x_{n}, x_{n+1}, x_{n+1}\right), G\left(x_{n}, x_{n+1}, x_{n+1}\right)\right\} \\
\leq \quad & a\left\{G\left(x_{n-1}, x_{n}, x_{n}\right)+G\left(x_{n}, x_{n+1}, x_{n+1}\right)\right\}+2 b G\left(x_{n}, x_{n+1}, x_{n+1}\right) \\
& +c\left\{G\left(x_{n-1}, x_{n}, x_{n}\right)+G\left(x_{n}, x_{n+1}, x_{n+1}\right)\right\}+d G\left(x_{n-1}, x_{n}, x_{n}\right) \\
& +e\left\{G\left(x_{n-1}, x_{n}, x_{n}\right)+G\left(x_{n}, x_{n+1}, x_{n+1}\right)\right\}
\end{aligned}
$$

which implies that,

$$
G\left(x_{n}, x_{n+1}, x_{n+1}\right) \leq \frac{a+c+d+e}{1-a-2 b-c-e} G\left(x_{n-1}, x_{n}, x_{n}\right)
$$

Let $q=\frac{a+c+d+e}{1-a-2 b-c-e}$, then $q<1$ since $2 a+2 b+2 c+d+2 e<1$ and by repeated application of $(6)$, we have

$$
G\left(x_{n}, x_{n+1}, x_{n+1}\right) \leq q^{n} G\left(x_{0}, x_{1}, x_{1}\right) .
$$

Then for all $n, m \in N, n<m$, we have by repeated use of the rectangle inequality and (7) that

$$
\begin{aligned}
G\left(x_{n}, x_{m}, x_{m}\right) \leq & G\left(x_{n}, x_{n+1}, x_{n+1}\right)+G\left(x_{n+1}, x_{n+2}, x_{n+2}\right) \\
& +G\left(x_{n+2}, x_{n+3}, x_{n+3}\right)+\cdots+G\left(x_{m-1}, x_{m}, x_{m}\right) \\
\leq & \left(q^{n}+q^{n+1}+\cdots+q^{m-1}\right) G\left(x_{0}, x_{1}, x_{1}\right) \\
\leq & \frac{q^{n}}{1-q} G\left(x_{0}, x_{1}, x_{1}\right) .
\end{aligned}
$$


Then, $\lim G\left(x_{n}, x_{m}, x_{m}\right)=0$, as $n, m \rightarrow \infty$, since $\lim \frac{q^{n}}{1-q} G\left(x_{0}, x_{1}, x_{1}\right)=0$, as $n, m \rightarrow \infty$. For $n, m, l \in N,\left(G_{5}\right)$ implies that

$$
G\left(x_{n}, x_{m}, x_{l}\right) \leq G\left(x_{n}, x_{m}, x_{m}\right)+G\left(x_{l}, x_{m}, x_{m}\right)
$$

taking limit as $n, m, l \rightarrow \infty$, we get $G\left(x_{n}, x_{m}, x_{l}\right) \rightarrow 0$. So $\left(x_{n}\right)$ is a $G$-Cauchy sequence. By completeness of $(X, G)$, there exists $u \in X$ such that $\left(x_{n}\right)$ is $G$-convergent to $u$. Suppose that $T(u) \neq u$, then

$$
\begin{aligned}
G\left(x_{n}, T(u), T(u)\right) \leq & a\left\{G\left(x_{n-1}, T(u), T(u)\right)+G\left(u, x_{n}, x_{n}\right)\right\} \\
& +b\{G(u, T(u), T(u))+G(u, T(u), T(u))\} \\
& +c\left\{G\left(u, x_{n}, x_{n}\right)+G\left(x_{n-1}, T(u), T(u)\right)\right\}+d G\left(x_{n-1}, u, u\right) \\
& +e \max \left\{G\left(x_{n-1}, x_{n}, x_{n}\right), G(u, T(u), T(u)), G(u, T(u), T(u))\right\} .
\end{aligned}
$$

Taking the limit as $n \rightarrow \infty$, and using the fact that the function $G$ is continuous on its variables, we have

$$
G(u, T(u), T(u)) \leq(a+2 b+c+e) G(u, T(u), T(u)),
$$

which is a contradiction since $0 \leq a+2 b+c+e<1$. So, $u=T(u)$.

For uniqueness of $u$, suppose that $v \neq u$ is such that $T(v)=v$, then (5) implies that

$$
\begin{aligned}
G(u, v, v)=G(T(u), T(v), T(v)) \leq & a\{G(u, v, v)+G(v, u, u)\}+2 b G(v, v, v) \\
& +c\{G(v, u, u)+G(u, v, v)\}+d G(u, v, v) \\
& +\operatorname{emax}\{G(u, u, u), G(v, v, v), G(v, v, v)\} \\
= & (a+c)\{G(u, v, v)+G(v, u, u)\}+d G(u, v, v) .
\end{aligned}
$$

So, it must be the case that,

$$
G(u, v, v) \leq \frac{a+c}{1-a-c-d} G(v, u, u) .
$$

Again by the same argument we will find

$$
G(v, u, u) \leq \frac{a+c}{1-a-c-d} G(u, v, v) .
$$

Thus, we have

$$
G(u, v, v) \leq\left(\frac{a+c}{1-a-c-d}\right)^{2} G(u, v, v)
$$


which implies that, $u=v$, since $0 \leq \frac{a+c}{1-a-c-d}<1$.

To see that $T$ is $G$-continuous at $u$, let $\left(y_{n}\right)$ be any sequence in $X$ such that $\left(y_{n}\right)$ is $G$-convergent to $u$. Then

$$
\begin{aligned}
G\left(T\left(y_{n}\right), T(u), T(u)\right) \leq & a\left\{G\left(y_{n}, T(u), T(u)\right)+G\left(u, T\left(y_{n}\right), T\left(y_{n}\right)\right)\right\} \\
& +b\{G(u, T(u), T(u))+G(u, T(u), T(u))\} \\
& +c\left\{G\left(u, T\left(y_{n}\right), T\left(y_{n}\right)\right)+G\left(y_{n}, T(u), T(u)\right)\right\}+d G\left(y_{n}, u, u\right) \\
& +e \max \left\{G\left(y_{n}, T\left(y_{n}\right), T\left(y_{n}\right)\right), G(u, T(u), T(u)), G(u, T(u), T(u))\right\}
\end{aligned}
$$

which gives,

$$
\begin{aligned}
G\left(T\left(y_{n}\right), u, u\right) \leq & a\left\{G\left(y_{n}, u, u\right)+2 G\left(T\left(y_{n}\right), u, u\right)\right\}+2 b G(u, u, u) \\
& +c\left\{2 G\left(T\left(y_{n}\right), u, u\right)+G\left(y_{n}, u, u\right)\right\}+d G\left(y_{n}, u, u\right) \\
& \left.+e\left\{G\left(y_{n}, u, u\right)+2 G\left(T\left(y_{n}\right), u, u\right)\right)\right\} .
\end{aligned}
$$

Thus, we deduce that,

$$
G\left(T\left(y_{n}\right), u, u\right) \leq \frac{a+c+d+e}{1-2 a-2 c-2 e} G\left(y_{n}, u, u\right) .
$$

Taking the limit as $n \rightarrow \infty$, we see that $G\left(T\left(y_{n}\right), u, u\right) \rightarrow 0$ and so, by Proposition 2.5, the sequence $\left(T\left(y_{n}\right)\right)$ is $G$-convergent to $u=T(u)$. Therefore Proposition 2.9 implies that $T$ is $G$-continuous at $u$.

We see that Theorem 3.4 is generalization of the Result [10, Theorem 2.9], where $[10$, Theorem 2.9] obtained by taking $b=c=d=e=0$ in Theorem 3.4 .

As an application of Theorem 3.4, we have the following results.

Corollary 3.5. Let $(X, G)$ be a complete $G$-metric space. Suppose that $T: X \longrightarrow X$ satisfies for some $m \in N$ :

$$
\begin{aligned}
G\left(T^{m}(x), T^{m}(y), T^{m}(z)\right) \leq & a\left\{G\left(x, T^{m}(y), T^{m}(y)\right)+G\left(y, T^{m}(x), T^{m}(x)\right)\right\} \\
& +b\left\{G\left(y, T^{m}(z), T^{m}(z)\right)+G\left(z, T^{m}(y), T^{m}(y)\right)\right\} \\
& +c\left\{G\left(z, T^{m}(x), T^{m}(x)\right)+G\left(x, T^{m}(z), T^{m}(z)\right)\right\} \\
& +d G(x, y, z)+\operatorname{emax}\left\{\begin{array}{c}
G\left(x, T^{m}(x), T^{m}(x)\right), \\
G\left(y, T^{m}(y), T^{m}(y)\right), \\
G\left(z, T^{m}(z), T^{m}(z)\right)
\end{array}\right\}
\end{aligned}
$$


for all $x, y, z \in X$, where $a, b, c, d, e \geq 0$ with $2 a+2 b+2 c+d+2 e<1$. Then $T$ has a unique fixed point (say $u$ ) in $X$ and $T^{m}$ is $G$-continuous at $u$.

Proof. The proof follows from Theorem 3.4 and the same argument used in Corollary 3.2.

Corollary 3.6. (see [13]) Let $X$ be a complete G-metric space. Suppose there is $k \in[0,1)$ such that the map $T: X \rightarrow X$ satisfies

$$
G(T(x), T(y), T(z)) \leq k G(x, y, z),
$$

for all $x, y, z \in X$. Then $T$ has a unique fixed point (say u) and $T$ is $G$ continuous at $u$.

Proof. Result follows from Theorem 3.4 by taking $a=b=c=e=0$ and $d=$ $k$.

Theorem 3.7. Let $(X, G)$ be a complete $G$-metric space, and let $T: X \longrightarrow$ $X$ be a mapping which satisfies the following condition

$G(T(x), T(y), T(z)) \leq k \max \left\{\begin{array}{c}G(x, T(x), T(x)), G(y, T(y), T(y)), G(z, T(z), T(z)), \\ G(x, T(y), T(y)), G(y, T(z), T(z)), G(z, T(x), T(x)), \\ G(x, T(z), T(z)), G(y, T(x), T(x)), G(z, T(y), T(y)), \\ G(x, T(y), T(z)), G(y, T(z), T(x)), G(z, T(x), T(y)), \\ G(x, y, T(z)), G(y, z, T(x)), G(z, x, T(y)), G(x, y, z)\end{array}\right\}$

for all $x, y, z \in X$, and $0 \leq k<\frac{1}{3}$. Then $T$ has a unique fixed point (say u) in $X$ and $T$ is $G$-continuous at $u$.

Proof. Let $x_{0} \in X$ be an arbitrary point and define the sequence $\left(x_{n}\right)$ by 
$x_{n}=T^{n}\left(x_{0}\right)$. Assume $x_{n} \neq x_{n+1}$ for all $n$. Then by (8), we have

$$
G\left(x_{n}, x_{n+1}, x_{n+1}\right) \leq k \max \left\{\begin{array}{l}
G\left(x_{n-1}, x_{n}, x_{n}\right), G\left(x_{n}, x_{n+1}, x_{n+1}\right), G\left(x_{n}, x_{n+1}, x_{n+1}\right), \\
G\left(x_{n-1}, x_{n+1}, x_{n+1}\right), G\left(x_{n}, x_{n+1}, x_{n+1}\right), G\left(x_{n}, x_{n}, x_{n}\right), \\
G\left(x_{n-1}, x_{n+1}, x_{n+1}\right), G\left(x_{n}, x_{n}, x_{n}\right), G\left(x_{n}, x_{n+1}, x_{n+1}\right), \\
G\left(x_{n-1}, x_{n+1}, x_{n+1}\right), G\left(x_{n}, x_{n+1}, x_{n+1}\right), G\left(x_{n}, x_{n}, x_{n+1}\right), \\
G\left(x_{n-1}, x_{n}, x_{n+1}\right), G\left(x_{n}, x_{n}, x_{n}\right), G\left(x_{n}, x_{n-1}, x_{n+1}\right), \\
G\left(x_{n-1}, x_{n}, x_{n}\right)
\end{array}\right\} .
$$

So,

$$
G\left(x_{n}, x_{n+1}, x_{n+1}\right) \leq k \max \left\{\begin{array}{l}
G\left(x_{n-1}, x_{n}, x_{n}\right), G\left(x_{n-1}, x_{n+1}, x_{n+1}\right), \\
G\left(x_{n}, x_{n}, x_{n+1}\right), G\left(x_{n-1}, x_{n}, x_{n+1}\right)
\end{array}\right\} .
$$

But by $\left(G_{5}\right)$, we have

$$
\begin{aligned}
G\left(x_{n-1}, x_{n}, x_{n+1}\right) & \leq G\left(x_{n-1}, x_{n}, x_{n}\right)+G\left(x_{n}, x_{n}, x_{n+1}\right) \\
& \leq G\left(x_{n-1}, x_{n}, x_{n}\right)+2 G\left(x_{n}, x_{n+1}, x_{n+1}\right)
\end{aligned}
$$

and

$$
G\left(x_{n-1}, x_{n+1}, x_{n+1}\right) \leq G\left(x_{n-1}, x_{n}, x_{n}\right)+G\left(x_{n}, x_{n+1}, x_{n+1}\right) .
$$

So, (9) becomes

$$
G\left(x_{n}, x_{n+1}, x_{n+1}\right) \leq k\left\{G\left(x_{n-1}, x_{n}, x_{n}\right)+2 G\left(x_{n}, x_{n+1}, x_{n+1}\right)\right\}
$$

which implies that,

$$
G\left(x_{n}, x_{n+1}, x_{n+1}\right) \leq \frac{k}{1-2 k} G\left(x_{n-1}, x_{n}, x_{n}\right)
$$

Let $q=\frac{k}{1-2 k}$, then $q<1$ since $0 \leq k<\frac{1}{3}$ and by repeated application of (10), we have

$$
G\left(x_{n}, x_{n+1}, x_{n+1}\right) \leq q^{n} G\left(x_{0}, x_{1}, x_{1}\right) .
$$


Then for all $n, m \in N, n<m$, we have by repeated use of the rectangle inequality and (11) that

$$
\begin{aligned}
G\left(x_{n}, x_{m}, x_{m}\right) \leq & G\left(x_{n}, x_{n+1}, x_{n+1}\right)+G\left(x_{n+1}, x_{n+2}, x_{n+2}\right) \\
& +G\left(x_{n+2}, x_{n+3}, x_{n+3}\right)+\cdots+G\left(x_{m-1}, x_{m}, x_{m}\right) \\
\leq & \left(q^{n}+q^{n+1}+\cdots+q^{m-1}\right) G\left(x_{0}, x_{1}, x_{1}\right) \\
\leq & \frac{q^{n}}{1-q} G\left(x_{0}, x_{1}, x_{1}\right) .
\end{aligned}
$$

Then, $\lim G\left(x_{n}, x_{m}, x_{m}\right)=0$, as $n, m \rightarrow \infty$, since $\lim \frac{q^{n}}{1-q} G\left(x_{0}, x_{1}, x_{1}\right)=0$, as $n, m \rightarrow \infty$. For $n, m, l \in N,\left(G_{5}\right)$ implies that

$$
G\left(x_{n}, x_{m}, x_{l}\right) \leq G\left(x_{n}, x_{m}, x_{m}\right)+G\left(x_{l}, x_{m}, x_{m}\right),
$$

taking limit as $n, m, l \rightarrow \infty$, we get $G\left(x_{n}, x_{m}, x_{l}\right) \rightarrow 0$. So $\left(x_{n}\right)$ is a $G$-Cauchy sequence. By completeness of $(X, G)$, there exists $u \in X$ such that $\left(x_{n}\right)$ is $G$-convergent to $u$. Suppose that $T(u) \neq u$, then $G\left(x_{n}, T(u), T(u)\right) \leq k \max \left\{\begin{array}{l}G\left(x_{n-1}, x_{n}, x_{n}\right), G(u, T(u), T(u)), G(u, T(u), T(u)), \\ G\left(x_{n-1}, T(u), T(u)\right), G(u, T(u), T(u)), G\left(u, x_{n}, x_{n}\right), \\ G\left(x_{n-1}, T(u), T(u)\right), G\left(u, x_{n}, x_{n}\right), G(u, T(u), T(u)), \\ G\left(x_{n-1}, T(u), T(u)\right), G\left(u, T(u), x_{n}\right), G\left(u, x_{n}, T(u)\right), \\ G\left(x_{n-1}, u, T(u)\right), G\left(u, u, x_{n}\right), G\left(u, x_{n-1}, T(u)\right), \\ G\left(x_{n-1}, u, u\right)\end{array}\right\}$.

Taking the limit as $n \rightarrow \infty$, and using the fact that the function $G$ is continuous on its variables, we have

$$
\begin{aligned}
G(u, T(u), T(u)) & \leq k \max \{G(u, T(u), T(u)), G(u, T(u), u)\} \\
& \leq k \max \{G(u, T(u), T(u)), 2 G(u, T(u), T(u))\} \\
& =2 k \cdot G(u, T(u), T(u))
\end{aligned}
$$

which is a contradiction since $0 \leq k<\frac{1}{3}$. So, $u=T(u)$.

For uniqueness of $u$, suppose that $v \neq u$ is such that $T(v)=v$, then $(9)$ implies that

$$
G(u, v, v)=G(T(u), T(v), T(v)) \leq k \max \{G(u, v, v), G(v, u, u)\} .
$$


So, it must be the case that,

$$
G(u, v, v) \leq k G(v, u, u) .
$$

Again by the same argument we will find

$$
G(v, u, u) \leq k G(u, v, v) .
$$

Thus, we have

$$
G(u, v, v) \leq k^{2} G(u, v, v)
$$

which implies that, $u=v$, since $0 \leq k<\frac{1}{3}$.

To see that $T$ is $G$-continuous at $u$, let $\left(y_{n}\right)$ be any sequence in $X$ such that $\left(y_{n}\right)$ is $G$-convergent to $u$. Then

$$
G\left(T\left(y_{n}\right), T(u), T(u)\right) \leq k \max \left\{\begin{array}{l}
G\left(y_{n}, T\left(y_{n}\right), T\left(y_{n}\right)\right), G(u, u, u), G(u, u, u), \\
G\left(y_{n}, u, u\right), G(u, u, u), G\left(u, T\left(y_{n}\right), T\left(y_{n}\right)\right), \\
G\left(y_{n}, u, u\right), G\left(u, T\left(y_{n}\right), T\left(y_{n}\right)\right), G(u, u, u), \\
G\left(y_{n}, u, u\right), G\left(u, u, T\left(y_{n}\right)\right), G\left(u, T\left(y_{n}\right), u\right), \\
G\left(y_{n}, u, u\right), G\left(u, u, T\left(y_{n}\right)\right), G\left(u, y_{n}, u\right), G\left(y_{n}, u, u\right)
\end{array}\right\}
$$

which gives,

$G\left(T\left(y_{n}\right), u, u\right) \leq k \max \left\{G\left(y_{n}, T\left(y_{n}\right), T\left(y_{n}\right)\right), G\left(u, T\left(y_{n}\right), T\left(y_{n}\right)\right), G\left(y_{n}, u, u\right)\right\}$.

By $\left(G_{5}\right)$, we have

$$
G\left(y_{n}, T\left(y_{n}\right), T\left(y_{n}\right)\right) \leq G\left(y_{n}, u, u\right)+G\left(u, T\left(y_{n}\right), T\left(y_{n}\right)\right) .
$$

Thus, we deduce that,

$$
G\left(T\left(y_{n}\right), u, u\right) \leq k\left\{G\left(y_{n}, u, u\right)+G\left(u, T\left(y_{n}\right), T\left(y_{n}\right)\right)\right\}
$$

So,

$$
G\left(T\left(y_{n}\right), u, u\right) \leq \frac{k}{1-2 k} G\left(y_{n}, u, u\right) .
$$

Taking the limit as $n \rightarrow \infty$, we see that $G\left(T\left(y_{n}\right), u, u\right) \rightarrow 0$ and so, by Proposition 2.5, the sequence $\left(T\left(y_{n}\right)\right)$ is $G$-convergent to $u=T(u)$. Therefore Proposition 2.9 implies that $T$ is $G$-continuous at $u$. 
As an application of Theorem 3.7, we have the following result.

Corollary 3.8. Let $(X, G)$ be a complete $G$-metric space, and let $T: X \longrightarrow$ $X$ be a mapping which satisfies the following condition for some $m \in N$ :

$$
G\left(T^{m}(x), T^{m}(y), T^{m}(z)\right) \leq k \max \left\{\begin{array}{l}
G\left(x, T^{m}(x), T^{m}(x)\right), G\left(y, T^{m}(y), T^{m}(y)\right), \\
G\left(z, T^{m}(z), T^{m}(z)\right), G\left(x, T^{m}(y), T^{m}(y)\right), \\
G\left(y, T^{m}(z), T^{m}(z)\right), G\left(z, T^{m}(x), T^{m}(x)\right), \\
G\left(x, T^{m}(z), T^{m}(z)\right), G\left(y, T^{m}(x), T^{m}(x)\right), \\
G\left(z, T^{m}(y), T^{m}(y)\right), G\left(x, T^{m}(y), T^{m}(z)\right), \\
G\left(y, T^{m}(z), T^{m}(x)\right), G\left(z, T^{m}(x), T^{m}(y)\right), \\
G\left(x, y, T^{m}(z)\right), G\left(y, z, T^{m}(x)\right), \\
G\left(z, x, T^{m}(y)\right), G(x, y, z)
\end{array}\right\}
$$

for all $x, y, z \in X$, and $0 \leq k<\frac{1}{3}$. Then $T$ has a unique fixed point (say $u$ ) in $X$ and $T^{m}$ is $G$-continuous at $u$.

Proof. The proof follows from the previous theorem and the same argument used in Corollary 3.2.

Theorem 3.9. Let $(X, G)$ be a complete $G$-metric space, and let $T: X \longrightarrow$ $X$ be a mapping which satisfies the following condition

$$
G(T(x), T(y), T(z)) \leq k \max \left\{\begin{array}{l}
G(x, T(y), T(y))+G(y, T(x), T(x))+G(z, T(z), T(z)), \\
G(y, T(z), T(z))+G(z, T(y), T(y))+G(x, T(x), T(x)), \\
G(z, T(x), T(x))+G(x, T(z), T(z))+G(y, T(y), T(y))
\end{array}\right\}
$$

for all $x, y, z \in X$, and $0 \leq k<\frac{1}{3}$. Then $T$ has a unique fixed point (say $u$ ) in $X$ and $T$ is $G$-continuous at $u$.

Proof. Let $x_{0} \in X$ be an arbitrary point and define the sequence $\left(x_{n}\right)$ by 
$x_{n}=T^{n}\left(x_{0}\right)$. Assume $x_{n} \neq x_{n+1}$ for all $n$. Then by (12), we get

$G\left(x_{n}, x_{n+1}, x_{n+1}\right) \leq k \max \left\{\begin{array}{l}G\left(x_{n-1}, x_{n+1}, x_{n+1}\right)+G\left(x_{n}, x_{n}, x_{n}\right)+G\left(x_{n}, x_{n+1}, x_{n+1}\right), \\ G\left(x_{n}, x_{n+1}, x_{n+1}\right)+G\left(x_{n}, x_{n+1}, x_{n+1}\right)+G\left(x_{n-1}, x_{n}, x_{n}\right), \\ G\left(x_{n}, x_{n}, x_{n}\right)+G\left(x_{n-1}, x_{n+1}, x_{n+1}\right)+G\left(x_{n}, x_{n+1}, x_{n+1}\right)\end{array}\right\}$.

By $\left(G_{5}\right)$, we have

$$
G\left(x_{n-1}, x_{n+1}, x_{n+1}\right) \leq G\left(x_{n-1}, x_{n}, x_{n}\right)+G\left(x_{n}, x_{n+1}, x_{n+1}\right) .
$$

So, (13) becomes

$$
G\left(x_{n}, x_{n+1}, x_{n+1}\right) \leq k\left\{G\left(x_{n-1}, x_{n}, x_{n}\right)+2 G\left(x_{n}, x_{n+1}, x_{n+1}\right)\right\}
$$

which implies that,

$$
G\left(x_{n}, x_{n+1}, x_{n+1}\right) \leq \frac{k}{1-2 k} G\left(x_{n-1}, x_{n}, x_{n}\right) .
$$

Let $q=\frac{k}{1-2 k}$, then $q<1$ since $0 \leq k<\frac{1}{3}$ and by repeated application of (14), we have

$$
G\left(x_{n}, x_{n+1}, x_{n+1}\right) \leq q^{n} G\left(x_{0}, x_{1}, x_{1}\right) .
$$

Then for all $n, m \in N, n<m$, we have by repeated use of the rectangle inequality and (15) that

$$
\begin{aligned}
G\left(x_{n}, x_{m}, x_{m}\right) \leq & G\left(x_{n}, x_{n+1}, x_{n+1}\right)+G\left(x_{n+1}, x_{n+2}, x_{n+2}\right) \\
& +G\left(x_{n+2}, x_{n+3}, x_{n+3}\right)+\cdots+G\left(x_{m-1}, x_{m}, x_{m}\right) \\
\leq & \left(q^{n}+q^{n+1}+\cdots+q^{m-1}\right) G\left(x_{0}, x_{1}, x_{1}\right) \\
\leq & \frac{q^{n}}{1-q} G\left(x_{0}, x_{1}, x_{1}\right) .
\end{aligned}
$$

Then, $\lim G\left(x_{n}, x_{m}, x_{m}\right)=0$, as $n, m \rightarrow \infty$, since $\lim \frac{q^{n}}{1-q} G\left(x_{0}, x_{1}, x_{1}\right)=0$, as $n, m \rightarrow \infty$. For $n, m, l \in N,\left(G_{5}\right)$ implies that

$$
G\left(x_{n}, x_{m}, x_{l}\right) \leq G\left(x_{n}, x_{m}, x_{m}\right)+G\left(x_{l}, x_{m}, x_{m}\right),
$$

taking limit as $n, m, l \rightarrow \infty$, we get $G\left(x_{n}, x_{m}, x_{l}\right) \rightarrow 0$. So $\left(x_{n}\right)$ is a $G$-Cauchy sequence. By completeness of $(X, G)$, there exists $u \in X$ such that $\left(x_{n}\right)$ is $G$-convergent to $u$. Suppose that $T(u) \neq u$, then 
$G\left(x_{n}, T(u), T(u)\right) \leq k \max \left\{\begin{array}{c}G\left(x_{n-1}, T(u), T(u)\right)+G\left(u, x_{n}, x_{n}\right)+G(u, T(u), T(u)), \\ G(u, T(u), T(u))+G(u, T(u), T(u))+G\left(x_{n-1}, x_{n}, x_{n}\right), \\ G\left(u, x_{n}, x_{n}\right)+G\left(x_{n-1}, T(u), T(u)\right)+G(u, T(u), T(u))\end{array}\right\}$.

Taking the limit as $n \rightarrow \infty$, and using the fact that the function $G$ is continuous on its variables, we have

$$
G(u, T(u), T(u)) \leq 2 k G(u, T(u), T(u))
$$

which is a contradiction since $0 \leq k<\frac{1}{3}$. So, $u=T(u)$.

To prove uniqueness of $u$, suppose that $v \neq u$ is such that $T(v)=v$, then

$$
G(u, v, v) \leq k \max \left\{\begin{array}{c}
G(u, v, v)+G(v, u, u)+G(v, v, v), \\
G(v, v, v)+G(v, v, v)+G(u, u, u), \\
G(v, u, u)+G(u, v, v)+G(v, v, v)
\end{array}\right\} .
$$

So, it must be the case that,

$$
G(u, v, v) \leq k\{G(u, v, v)+G(v, u, u)\}
$$

which implies that,

$$
G(u, v, v) \leq \frac{k}{1-k} G(v, u, u) .
$$

Again by the same argument we will find

$$
G(v, u, u) \leq \frac{k}{1-k} G(u, v, v) .
$$

Thus, we deduce that,

$$
G(u, v, v) \leq\left(\frac{k}{1-k}\right)^{2} G(u, v, v)
$$

which implies that, $u=v$, since $0 \leq k<\frac{1}{3}$. 
To see that $T$ is $G$-continuous at $u$, let $\left(y_{n}\right)$ be any sequence in $X$ such that $\left(y_{n}\right)$ is $G$-convergent to $u$. Then

$$
G\left(T\left(y_{n}\right), T(u), T(u)\right) \leq k \max \left\{\begin{array}{l}
G\left(y_{n}, T(u), T(u)\right)+G\left(u, T\left(y_{n}\right), T\left(y_{n}\right)\right) \\
+G(u, T(u), T(u)), \\
G(u, T(u), T(u))+G(u, T(u), T(u)) \\
+G\left(y_{n}, T\left(y_{n}\right), T\left(y_{n}\right)\right), \\
G\left(u, T\left(y_{n}\right), T\left(y_{n}\right)\right)+G\left(y_{n}, T(u), T(u)\right) \\
+G(u, T(u), T(u))
\end{array}\right\}
$$

which gives,

$G\left(T\left(y_{n}\right), u, u\right) \leq k \max \left\{G\left(y_{n}, u, u\right)+G\left(u, T\left(y_{n}\right), T\left(y_{n}\right)\right), G\left(y_{n}, T\left(y_{n}\right), T\left(y_{n}\right)\right)\right\}$.

By $\left(G_{5}\right)$, we have

$$
G\left(y_{n}, T\left(y_{n}\right), T\left(y_{n}\right)\right) \leq G\left(y_{n}, u, u\right)+G\left(u, T\left(y_{n}\right), T\left(y_{n}\right)\right) .
$$

Thus, (16) becomes

$$
G\left(T\left(y_{n}\right), u, u\right) \leq k\left\{G\left(y_{n}, u, u\right)+G\left(u, T\left(y_{n}\right), T\left(y_{n}\right)\right)\right\} .
$$

Again by $\left(G_{5}\right)$, we have

$$
G\left(u, T\left(y_{n}\right), T\left(y_{n}\right)\right) \leq 2 G\left(T\left(y_{n}\right), u, u\right) .
$$

Therefore (17) implies that

$$
G\left(T\left(y_{n}\right), u, u\right) \leq k\left\{G\left(y_{n}, u, u\right)+2 G\left(T\left(y_{n}\right), u, u\right\}\right.
$$

and we deduce that

$$
G\left(T\left(y_{n}\right), u, u\right) \leq \frac{k}{1-2 k} G\left(y_{n}, u, u\right) .
$$

Taking the limit as $n \rightarrow \infty$, we see that $G\left(T\left(y_{n}\right), u, u\right) \rightarrow 0$ and so, by Proposition 2.5, the sequence $\left(T\left(y_{n}\right)\right)$ is $G$-convergent to $u=T(u)$. Therefore Proposition 2.9 implies that $T$ is $G$-continuous at $u$.

Some results in the literature related to Theorem 3.7 and Theorem 3.9 are available in [9]. 
Corollary 3.10. Let $(X, G)$ be a complete $G$-metric space, and let $T$ : $X \longrightarrow X$ be a mapping which satisfies the following condition for some $m \in N$ :

$G\left(T^{m}(x), T^{m}(y), T^{m}(z)\right) \leq k \max \left\{\begin{array}{l}G\left(x, T^{m}(y), T^{m}(y)\right)+G\left(y, T^{m}(x), T^{m}(x)\right) \\ +G\left(z, T^{m}(z), T^{m}(z)\right), \\ G\left(y, T^{m}(z), T^{m}(z)\right)+G\left(z, T^{m}(y), T^{m}(y)\right) \\ +G\left(x, T^{m}(x), T^{m}(x)\right), \\ G\left(z, T^{m}(x), T^{m}(x)\right)+G\left(x, T^{m}(z), T^{m}(z)\right) \\ +G\left(y, T^{m}(y), T^{m}(y)\right)\end{array}\right\}$

for all $x, y, z \in X$, and $0 \leq k<\frac{1}{3}$. Then $T$ has a unique fixed point (say $u$ ) in $X$ and $T^{m}$ is $G$-continuous at $u$.

Proof. The proof follows from the previous theorem and the same argument used in Corollary 3.2.

Theorem 3.11. Let $(X, G)$ be a complete $G$-metric space, and let $T$ : $X \longrightarrow X$ be a mapping which satisfies the following condition

$G(T(x), T(y), T(z)) \leq k \max \left\{\begin{array}{l}G(x, T(y), T(y))+G(y, T(x), T(x))+G(z, T(x), T(y)), \\ G(y, T(z), T(z))+G(z, T(y), T(y))+G(x, T(y), T(z)), \\ G(z, T(x), T(x))+G(x, T(z), T(z))+G(y, T(z), T(x))\end{array}\right\}$

for all $x, y, z \in X$, and $0 \leq k<\frac{1}{4}$. Then $T$ has a unique fixed point (say $u$ ) in $X$ and $T$ is $G$-continuous at $u$.

Proof. The proof follows from the argument similar to that used in Theorem 3.9 .

Theorem 3.12. Let $(X, G)$ be a complete $G$-metric space, and let $T$ : 
$X \longrightarrow X$ be a mapping which satisfies the following condition

$G(T(x), T(y), T(z)) \leq k \max \left\{\begin{array}{c}G(x, T(y), T(y))+G(y, T(x), T(x))+G(x, y, T(z)), \\ G(y, T(z), T(z))+G(z, T(y), T(y))+G(y, z, T(x)), \\ G(z, T(x), T(x))+G(x, T(z), T(z))+G(z, x, T(y))\end{array}\right\}$

for all $x, y, z \in X$, and $0 \leq k<\frac{1}{5}$. Then $T$ has a unique fixed point (say $u$ ) in $X$ and $T$ is $G$-continuous at $u$.

Proof. The proof can be obtained by the argument similar to that used in Theorem 3.9.

\section{References}

[1] R.Chugh, T.Kadian, A.Rani, and B.E.Rhoades, "Property $P$ in $G$-metric spaces," Fixed Point Theory and Applications, vol. 2010, Article ID 401684, 12 pages, 2010.

[2] B.C.Dhage, "Generalised metric spaces and mappings with fixed point," Bulletin of the Calcutta Mathematical Society, vol.84, no. 4, pp. 329-336, 1992.

[3] B.C.Dhage, "Generalised metric spaces and topological structure- I," Analele Stiintifice ale Universitătii "Al.I.Cuza" din Iasi. Serie Nouă. Matematică, vol.46, no. 1, pp. 3-24, 2000.

[4] S.Gähler, "2-metrische Räume und ihre topologische Struktur," Mathematische Nachrichten, vol.26, pp. 115-148, 1963.

[5] S.Gähler, "Zur geometric 2-metrische räume," Revue Roumaine de Mathématiques Pures et Appliquées, vol.40, pp. 664-669, 1966.

[6] K.S.Ha,Y.J.Cho, and A.White, "Strictly convex and strictly 2-convex 2normed spaces," Mathematica Japonica, vol.33, no. 3, pp. 375-384, 1988.

[7] Sushanta Kumar Mohanta and A.P.Baisnab, "A class of Ćirić operators and their fixed points," Bulletin of the Allahabad Mathematical Society, vol. 20, pp. 79-88, 2005.

[8] Z.Mustafa and B.Sims, "A new approach to generalized metric spaces," Journal of Nonlinear and convex Analysis, vol. 7, no. 2, pp. 289-297, 2006. 
[9] Z.Mustafa and B.Sims, "Fixed point theorems for contractive mappings in complete G-metric spaces," Fixed Point Theory and Applications, vol. 2009, Article ID 917175, 10 pages, 2009.

[10] Z.Mustafa, H.Obiedat, and F. Awawdeh, "Some fixed point theorem for mapping on complete G-metric spaces," Fixed Point Theory and Applications, vol. 2008, Article ID 189870, 12 pages, 2008.

[11] Z.Mustafa, W.Shatanawi, and M.Bataineh, "Existence of fixed point results in G-metric spaces," International Journal of Mathematics and Mathematical Sciences, vol. 2009, Article ID 283028, 10 pages, 2009.

[12] Z.Mustafa and B.Sims, "Some remarks concerning $D$-metric spaces," in Proceedings of the International Conference on Fixed Point Theory and Applications, pp. 189-198, Valencia, Spain, July 2004.

[13] Z.Mustafa, A new structure for generalized metric spaces-with applications to fixed point theory, Ph.D. thesis, The University of Newcastle, Callaghan, Australia, 2005.

[14] Z.Mustafa and H. Obiedat, "A fixed points theorem of Reich in G-metric spaces," Cubo A Mathematics Journal, vol. 12, no. 01, pp. 83-93, 2010.

[15] Z.Mustafa, F. Awawdeh and W.Shatanawi, "Fixed point theorem for expansive mappings in G-metric spaces," Int. J. Contemp. Math. Sciences, vol. 5, no. 50, pp. 2463-2472, 2010.

[16] S.V.R.Naidu, K.P.R.Rao, and N.Srinivasa Rao, "On the concept of balls in a D-metric space," International Journal of Mathematics and Mathematical Sciences, no. 1, pp. 133-141, 2005.

[17] W.Shatanawi," Fixed point theory for contractive mappings satisfying $\phi$ maps in G-metric spaces," Fixed Point Theory and Applications, vol. 2010, Article ID 181650, 9 pages, 2010.

Department of Mathematics,

West Bengal State University,

Barasat, 24 Parganas (North),

Kolkata 700126.

Email: smwbes@yahoo.in 
